\title{
Altered expression of microRNAs in the rat diaphragm in a model of ventilator- induced diaphragm dysfunction after controlled mechanical ventilation
}

\author{
Pengcheng Wang ${ }^{1,2}$, Xianlong Zhou ${ }^{1,2}$, Gang Li $i^{3}$ Haoli Ma ${ }^{3}$, Ruining Liu ${ }^{1,2}$ and Yan Zhao ${ }^{1,2^{*}}$ (D)
}

\begin{abstract}
Background: Ventilator-induced diaphragm dysfunction (VIDD) is a common complication of life support by mechanical ventilation observed in critical patients in clinical practice and may predispose patients to severe complications such as ventilator-associated pneumonia or ventilator discontinuation failure. To date, the alterations in microRNA (miRNA) expression in the rat diaphragm in a VIDD model have not been elucidated. This study was designed to identify these alterations in expression.

Results: Adult male Wistar rats received conventional controlled mechanical ventilation (CMV) or breathed spontaneously for $12 \mathrm{~h}$. Then, their diaphragm tissues were collected for RNA extraction. The miRNA expression alterations in diaphragm tissue were investigated by high-throughput microRNA-sequencing (miRNA-seq). For targeted mRNA functional analysis, gene ontology (GO) analyses and Kyoto Encyclopedia of Genes and Genomes (KEGG) pathway analyses were subsequently conducted. GRT-PCR validation and luciferase reporter assays were performed. We successfully constructed a model of ventilator-induced diaphragm dysfunction and identified 38 significantly differentially expressed (DE) miRNAs, among which 22 miRNAs were upregulated and 16 were downregulated. GO analyses identified functional genes, and KEGG pathway analyses revealed the signaling pathways that were most highly correlated, which were the MAPK pathway, FoxO pathway and Autophagy-animal. Luciferase reporter assays showed that STAT3 was a direct target of both miR-92a-1-5p and miR874-3p and that Trim63 was a direct target of miR-3571.
\end{abstract}

Conclusions: The current research supplied novel perspectives on miRNAs in the diaphragm, which may not only be implicated in diaphragm dysfunction pathogenesis but could also be considered as therapeutic targets in diaphragm dysfunction.

Keywords: Controlled mechanical ventilation, Ventilator-induced diaphragm dysfunction, microRNA, Rat

\footnotetext{
* Correspondence: doctoryanzhao@whu.edu.cn

'Emergency Center, Zhongnan Hospital of Wuhan University, 430071 Wuhan,

China

${ }^{2}$ Hubei Clinical Research Center for Emergency and Resuscitation, Zhongnan

Hospital of Wuhan University, 430071 Wuhan, China

Full list of author information is available at the end of the article
}

C C The Author(s). 2021 Open Access This article is licensed under a Creative Commons Attribution 4.0 International License, which permits use, sharing, adaptation, distribution and reproduction in any medium or format, as long as you give appropriate credit to the original author(s) and the source, provide a link to the Creative Commons licence, and indicate if changes were made. The images or other third party material in this article are included in the article's Creative Commons licence, unless indicated otherwise in a credit line to the material. If material is not included in the article's Creative Commons licence and your intended use is not permitted by statutory regulation or exceeds the permitted use, you will need to obtain permission directly from the copyright holder. To view a copy of this licence, visit http://creativecommons.org/licenses/by/4.0/. The Creative Commons Public Domain Dedication waiver (http://creativecommons.org/publicdomain/zero/1.0/) applies to the data made available in this article, unless otherwise stated in a credit line to the data. 


\section{Background}

Many critically ill patients such as those with haemorrhagic shock [1], sepsis [2], or respiratory failure [3], including patients with COVID-19 and dysponea, are treated with ventilation, and ventilation may rapidly induce diaphragm disuse, weakness and atrophy [4]. This pathogenic process has been designated ventilatorinduced diaphragm dysfunction (VIDD). Emerging evidence implies that it is the major complication of controlled mechanical ventilation (CMV). The development of VIDD is closely related to poor or adverse clinical prognoses in critically ill patients, who often suffer from an increased time of CMV, or even weaning failure.

The molecular and cellular mechanisms of VIDD have yet to be fully elucidated, and various studies of VIDD have linked it to autophagy [5], mitochondrial dysfunction [6], endoplasmic reticulum stress [7] and oxidative stress [8].

Recent research has focused on miRNAs, which are small endogenous RNA molecules with lengths of 19-23 nt. They bind to target sites (i.e., the 3'-UTR) in the noncoding regions of mRNA molecules to post transcriptionally regulate gene expression and protein synthesis. They are involved in the onset and pathology of many diseases. miRNAs have been reported to be involved in many diseases involving muscle atrophy. For instance, miR-21 was confirmed to be involved in skeletal muscle ageing and the decline in the potential for muscle regeneration [9]; miR-542-3p/5p was reported to affect patients with muscle atrophy via the acceleration of mitochondrial dysfunction [10]; and miR-182 was shown to be an important regulator of muscle atrophy-inducing genes under catabolic disease conditions [11]. It is clear that some miRNAs can regulate muscle atrophy in other pathogeneses; however, the actual effect of miRNAs in VIDD is not yet understood.

After CMV, accompanied by diaphragm dysfunction, the altered expression of diaphragm miRNAs might play a major role in modulating signaling pathways, gene expression and protein synthesis. All of the above factors might constitute a key mechanism of pathogenesis and be novel therapeutic targets.

The present study was aimed at idendifying differentially expressed miRNAs, finding novel therapeutic targets, and evaluating miRNA expression alterations in the rat diaphragm after CMV by high-throughput RNA sequencing. Various bioinformatic analyses were performed to predict miRNA functions according to Gene Ontologygene ontology (GO) analysis and Kyoto Encyclopedia of Gene and Genomes (KEGG) pathway analysis. A pathway relationship network was created based on the KEGG pathway analysis results and the identified DE (differential expressed) miRNAs to discover the relationships among them. Furthermore, nine
miRNAs were randomly selected to perform quantitative reverse transcription-polymerase chain reaction (qRTPCR) analysis, to validate the reliability of the RNA-seq results. Finally, a luciferase reporter assay was conducted to ascertain the links between miR-3571 and Trim63, miR-92a-1-5p and STAT3, and miR-874-3p and STAT3. This study revealed some novel and clear roles of these DE miRNAs in the diaphragm after CMV.

\section{Results \\ Differential expression of miRNAs in diaphragm tissue after VIDD}

miRNAs were detected in four CMV and four control diaphragm tissue samples. The miRNA-seq reads of all samples are shown in Figure S1. The heatmap shows the distances between the samples. The samples with higher similarity were clustered together with high priority (Fig. 1). We identified 38 significantly DE miRNAs according to the standard of a $p$ value $<0.05$ and $\left|\log _{2} \mathrm{FC}\right|>1$ in the expression level. Twenty-two of the 38 miRNAs were upregulated, and the other 16 were downregulated (Fig. 2A). A volcano plot was created based on the mean miRNA expression values in each group, and the threshold was set at 2.0 for the fold change of expression (Fig. 2B). A heatmap of 38 DE miRNAs was constructed to illustrate the discernible miRNA expression profiles (Fig. 2C). The DE miRNAs were listed in Table 1. Detailed information on the differentially expressed (DE) miRNAs $\left(\left|\log _{2} \mathrm{FC}\right|>1\right.$ and $\left.p<0.05\right)$ were listed in Table S2.

\section{GO analysis of DE miRNA-targeted genes}

The identified genes (mRNAs) were subjected to GO functional analysis to explain and hypothesize the functions of the DE miRNAs (Fig. 3A). These GO terms applied a correction for multiple testing. The type of correction was Benjamini and Hochberg (BH) of False Discovery Rate (FDR). Detailed information on the differentially enriched GO analysis is listed in Table S3. The results of GO analysis can be classified into three major categories: biological process (BP), cellular component (CC), and molecular function (MF). These columns are presented in the figure in descending order of $-\log _{10} p$ values. The $\mathrm{BP}$ results showed that the $\mathrm{DE}$ miRNAs were strongly related to the regulation of histamine secretion by mast cells, transcription by RNA polymerase II, positive regulation of transcription by RNA polymerase II, and so on. Among the GO CC terms that were significantly enriched in DE miRNAs, the miRNAs were associated with the clathrin-coated endocytic vesicle membrane, nucleus, cytoplasm, cytosol, cell junction. In the MF category, the DE miRNAs were associated with terms such as protein binding, protein kinase binding. The distribution of DE miRNA target genes at GO level2 is shown in Figure S2A. 


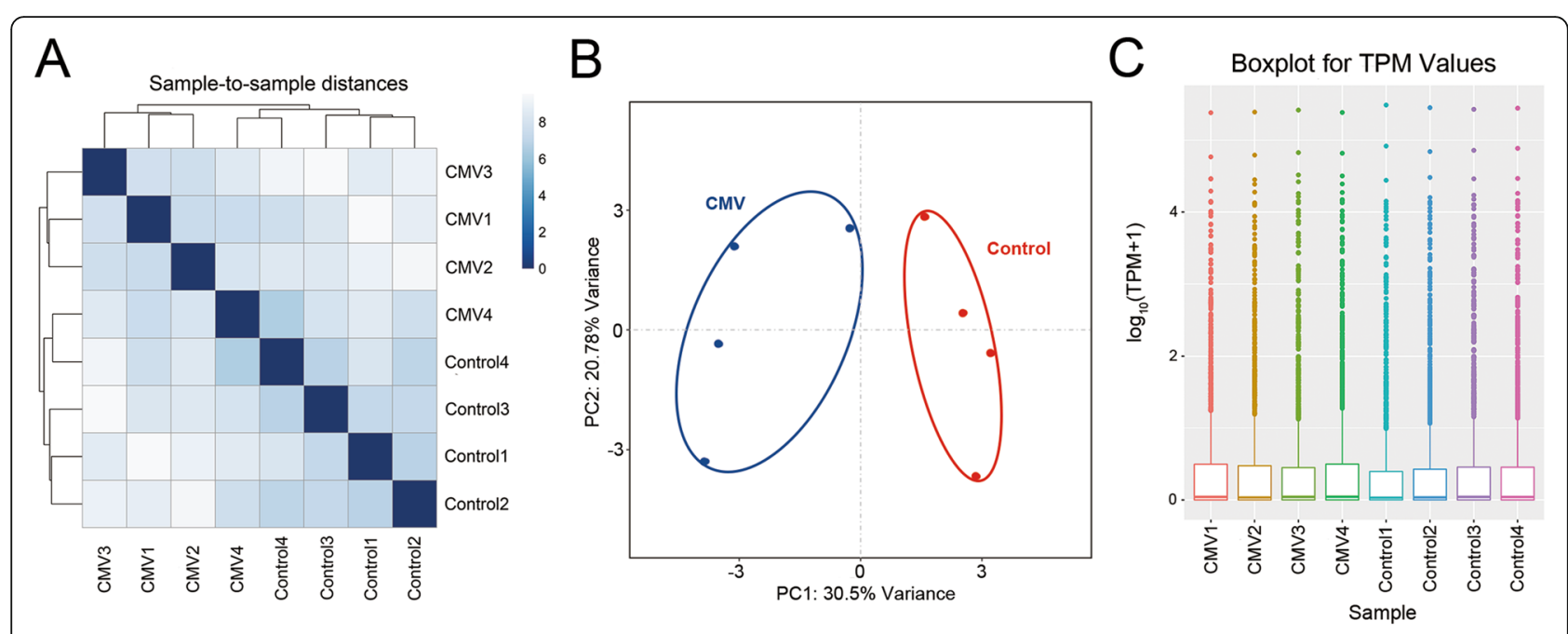

Fig. 1 Correlations of samples. A The heatmap shows the sample-to-sample distance, and samples with high similarity were preferentially clustered together. B PCA analyses. C Boxplot of sample TPM values

KEGG pathway analysis of DE miRNA-targeted genes The dot plot of the identified KEGG pathways shows the top 20 significantly enriched pathways in order of enrichment score values. These KEGG terms applied a correction for multiple testing. The type of correction was Benjamini and Hochberg (BH) of False Discovery Rate (FDR). Detailed information on the differentially enriched KEGG pathways is listed in Table S4. The pathways influenced by the variation in miRNAs and targeted mRNAs in diaphragm tissue after VIDD could be predicted. The top 20 pathways are showed in Fig. 3B, and included the Oocyte meiosis, MAPK signaling pathway, Protein processing in endoplasmic reticulum, GnRH signaling pathway, Cellular senescence, Autophagy-animal.

According to the KEGG classification, the numbers of proteins or genes identified at different functional levels, including cellular processes, environmental information processing, genetic information processing, human diseases, metabolism and organismal systems, were



\section{B}

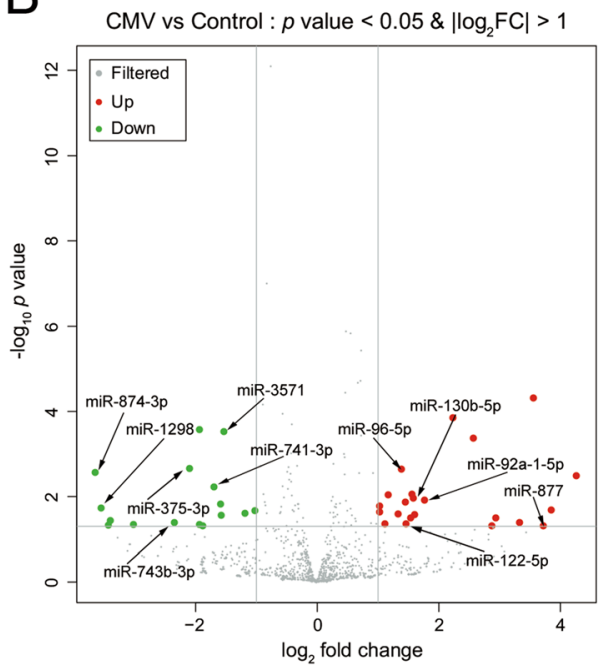

C CMV vs Control: $p$ value $<0.05 \&\left|\log _{2} \mathrm{FC}\right|>1$

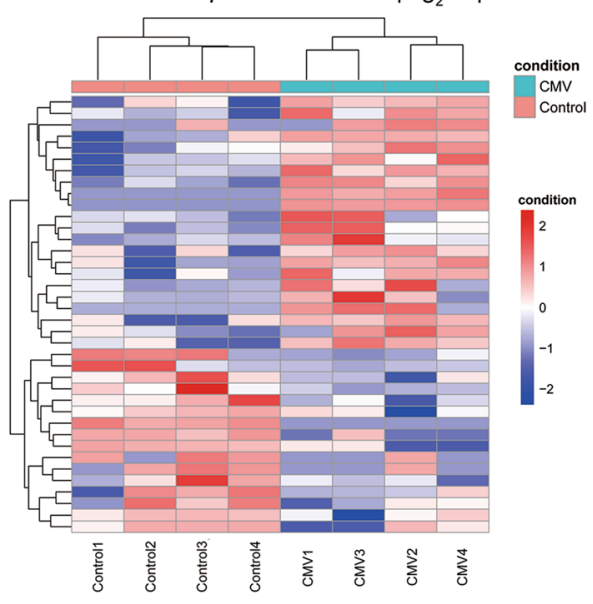

Fig. 2 Differential expression of microribonucleic acids (miRNAs) in diaphragm tissue after mechanical ventilation. A Statistics of DE miRNAs. B Volcano plot of miRNA expression levels. The red dots indicate upregulated miRNAs with significantly differential expression, the green dots indicate downregulated miRNAs with significantly differential expression, and the grey dots indicate miRNAs without differential expression. The $X$-axis shows the $\log _{2}$ fold change values; the $Y$-axis shows the $-\log _{10} p$ values. $C$ Heat map of DE miRNAs. Each row represents one probe set; each column represents one single sample. The dendrogram at the top reveals sample clustering; the dendrogram to the left reveals gene clustering 
Table 1 The differentially expressed miRNAs

\begin{tabular}{|c|c|c|c|c|c|}
\hline miRNA ID & Log2FC & $p$-value & up/down & Sequence & Length \\
\hline novel10_mature & 2.585894365 & 0.000420713 & Up & TCAGTGTGCTAGAGTCCTCGAAGA & 24 \\
\hline novel10_star & 3.578370892 & 4.88E-05 & Up & TCCGAGGCTAGAGTCACGCTC & 21 \\
\hline novel179_mature & 2.886092162 & 0.048552039 & Up & ATCTCGGTGGGACCGCCA & 18 \\
\hline novel214_mature & 1.572344804 & 0.008723536 & Up & ACTGGACTTGGAGTCAGAAGA & 21 \\
\hline novel231_mature & -3.001989158 & 0.04517486 & Down & TTGGTACCTGTTTCCTGTIT & 20 \\
\hline novel237_mature & 2.953174649 & 0.031303233 & Up & CGTGACTGTACCTGGTATT & 19 \\
\hline novel30_mature & 1.558144602 & 0.032272673 & Up & ATATAGAGGATAATATAAATGT & 22 \\
\hline novel316_mature & 1.470591476 & 0.013690233 & Up & AAAGTTTAACTTCTGCCA & 18 \\
\hline novel31_mature & -1.566475963 & 0.027139728 & Down & TATGGCGCTCCTCTGAGTAGA & 21 \\
\hline novel322_mature & 3.342200406 & 0.041283283 & Up & TGACCCTCCTTTGCTCCTCAGG & 22 \\
\hline novel379_mature & -3.384012887 & 0.037334425 & Down & TCTCCGGCCTCTCGCGGGACCT & 22 \\
\hline novel402_mature & 1.13285745 & 0.043958532 & Up & TCTGCTGACTGCCCATGGA & 19 \\
\hline novel427_mature & 2.252020331 & 0.000142047 & Up & TCCGGCTGCGTCGGGCGTG & 19 \\
\hline novel428_mature & 3.867171168 & 0.020676937 & Up & CCTGGGGCGGGCTGTGGGCTGTC & 23 \\
\hline novel458_mature & -1.922705588 & 0.000263353 & Down & CACTGGACTTGGAGTCAGAAGA & 22 \\
\hline novel462_mature & 4.274081175 & 0.003197987 & Up & TTTGGTCTAAGGCTGGAACTT & 22 \\
\hline novel566_mature & 1.045979075 & 0.022908305 & Up & CATGGACGGTGTGAGGCCA & 19 \\
\hline novel73_mature & -3.424160743 & 0.04782349 & Down & TAAACCAGTCAGAGGATGGTAGG & 23 \\
\hline novel75_mature & 1.622119991 & 0.026499961 & Up & ATGTAGTACTAAGTCTGTCACG & 22 \\
\hline rno-miR-122-5p & 1.47954028 & 0.04375855 & Up & TGGAGTGTGACAATGGTGTTG & 22 \\
\hline rno-miR-1298 & -3.537711659 & 0.018835052 & Down & TTCATTCGGCTGTCCAGATGTA & 22 \\
\hline rno-miR-130b-5p & 1.596831274 & 0.010734419 & Up & ACTCTTTCCCTGTTGCACTACT & 22 \\
\hline rno-miR-147 & 1.347240696 & 0.025313554 & Up & GTGTGCGGAAATGCTTCTGCTA & 22 \\
\hline rno-miR-183-5p & 1.047179965 & 0.016657263 & Up & TATGGCACTGGTAGAATTCACT & 22 \\
\hline rno-miR-200a-5p & -1.862942448 & 0.049359836 & Down & CATCTTACCGGACAGTGCTGG & 21 \\
\hline rno-miR-200c-3p & -1.572397551 & 0.014783046 & Down & TAATACTGCCGGGTAATGATG & 21 \\
\hline rno-miR-208b-3p & 1.183001365 & 0.009169665 & Up & ATAAGACGAACAAAAGGT & 18 \\
\hline rno-miR-296-3p & -1.170361668 & 0.024842243 & Down & GAGGGTTGGGTGGAGGCTCTCC & 22 \\
\hline rno-miR-3571 & -1.516247884 & 0.00029504 & Down & TACACACTTCTTTACATTCCATA & 23 \\
\hline rno-miR-375-3p & -2.086674249 & 0.002167474 & Down & TTTGTCGTTCGGCTCGCGTGA & 22 \\
\hline rno-miR-377-5p & -1.005516248 & 0.021749086 & Down & AGAGGTTGCCCTTGGTGAATTC & 22 \\
\hline rno-miR-501-5p & -1.925263887 & 0.045437012 & Down & AATCCTTTGTCCCTGGGTGA & 20 \\
\hline rno-miR-741-3p & -1.685014571 & 0.005957175 & Down & AAAGATGCCACGCTATGTAGAT & 22 \\
\hline rno-miR-743b-3p & -2.333443622 & 0.040744086 & Down & GAAAGACACCATACTGAATAGA & 22 \\
\hline rno-miR-874-3p & -3.638678583 & 0.002704144 & Down & CTGCCCTGGCCCGAGGGACCGA & 22 \\
\hline rno-miR-877 & 3.735698614 & 0.048157151 & Up & GTAGAGGAGATGGCGCAGGG & 20 \\
\hline rno-miR-92a-1-5p & 1.784733476 & 0.012196833 & Up & AGGTTGGGATTTGTCGCAATGCT & 23 \\
\hline rno-miR-96-5p & 1.398245576 & 0.002265536 & Up & TITGGCACTAGCACATTITGCT & 23 \\
\hline
\end{tabular}

FC fold change

quantified. The signal transduction of environmental information processing category appeared to include the highest percentage of all identified genes, which may imply a major role in VIDD pathogenesis. The categories with the next highest percentages of the identified genes were infectious diseases and cancers of Human Diseases, Endocrine system of Organismal Systems. The relatively high percentages if genes in these categories suggested that they play important roles in this pathogenic process. (Figure S2B). 
A

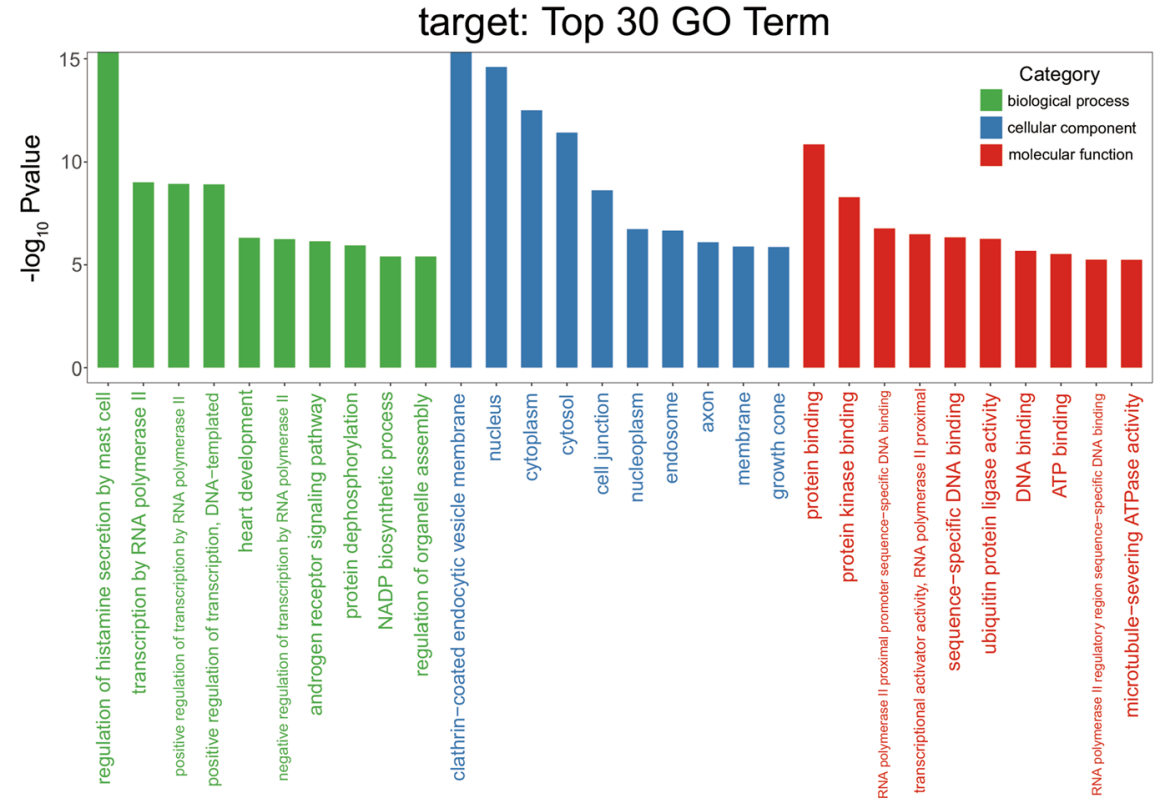

B

target: KEGG Enrichment top 20



Fig. 3 Gene Ontology (GO) analysis and Kyoto Encyclopedia of Genes and Genomes (KEGG) pathway analysis. A GO analysis of DE miRNAs with the top 30 genes according to the $-\log _{10} p$ value for each GO term. B KEGG pathway analysis of differentially expressed miRNAs, with the top 20 enrichment scores

The pathway relationship network of the top 20 pathways enriched in DE miRNAs was drawn (Fig. 4). This network implied that among all the pathways, three particular pathways may have an upstream regulatory effect. These pathways were the MAPK signaling pathway, the FoxO signaling pathway and Autophagy-animal pathway.

\section{Validation of the reliability of miRNA-seq data by qRT- PCR}

Nine DE miRNAs were randomly selected to verify the accuracy of miRNA-seq results. U6 was set as an internal control. Comparisons between the expression levels of these miRNAs determined by miRNA-seq or qRT-PCR are shown in Fig. 5. The expression levels 


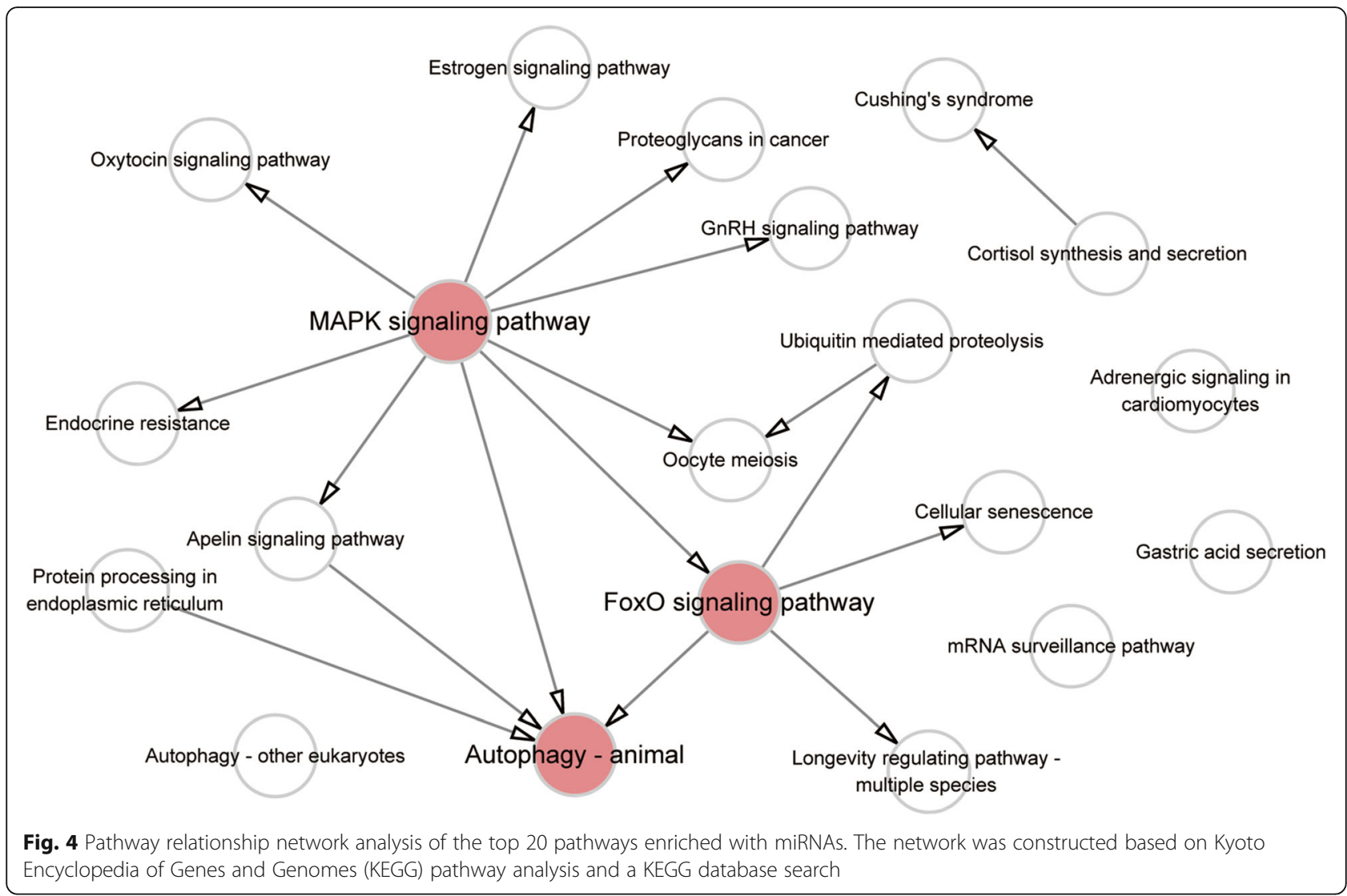

revealed by qRT-PCR analysis were calculated by the $2^{-\Delta \Delta C T}$ method. The data were expressed as mean \pm SD values. The results were significant for seven of the nine miRNAs $(p<0.05)$. The variation trends of miR-296-3p, miR-3571, miR-375-3p, miR-377-5p, and miR-743b-3p were in accordance with the miRNA sequencing data, while those of the two other miRNA-miR-183-5p and miR-208b-3p-were not. Although the differences were nonsignificant ( $p$ value $>0.05$ ), the results for two other miRNAs (miR-122-5p and miR-741-3p) implied concordance with the miRNA-seq results (data not shown). In total, the verifiable validation rate was $7 / 9$. This validation result showed that the miRNA-seq results were reliable.

\section{Luciferase validation}

MicroRNAs exert their inhibitory effects on mRNAs by binding to their 3'-UTRs. Multiple direct targets of miRNAs have been described in the literature to date. We searched for novel targets of miRNAs within mRNAs that could potentially account for the variation in the expression of miRNAs in diaphragm tissue after the rats were subjected to controlled mechanical ventilation. On the basis of bioinformatic analysis in the miRDB (http:// mirdb.org), TargetScan (http://www.targetscan.org) and Diana (http://diana.imis.athena-innovation.gr/
DianaTools) databases as well as previous studies, we selected three miRNA-mRNA interactions for further analysis and tested them by using luciferase reporter assays. We separately cloned the 3'-UTRs of Trim63 and STAT3 containing miR binding sites, downstream of the luciferase reporter gene. The normalized luciferase activity was significantly reduced upon cotransfection with miR-3571 and Trim63 WT and upon cotransfection with either miR-92a-1-5p or miR-874-3p and STAT3 WT. The cells that were cotransfected with MUT and either mimic-NC or mimic miRNA showed no significant differences in each group (Fig. 6). These results indicate that STAT3 is a direct target of both miR-92a-1$5 p$ and miR-874-3p and that Trim63 is a direct target of miR-3571.

\section{Discussion}

In the present study, we revealed the altered expression profile of miRNAs in the rat diaphragm tissue after CMV for the first time by high-throughput miRNAome sequencing followed by GO and KEGG pathway analysis. We made several novel findings which may accelerate the understanding of post-CMV molecular and intermolecular alterations coupled with the critical signaling pathways in which these molecules participate. 


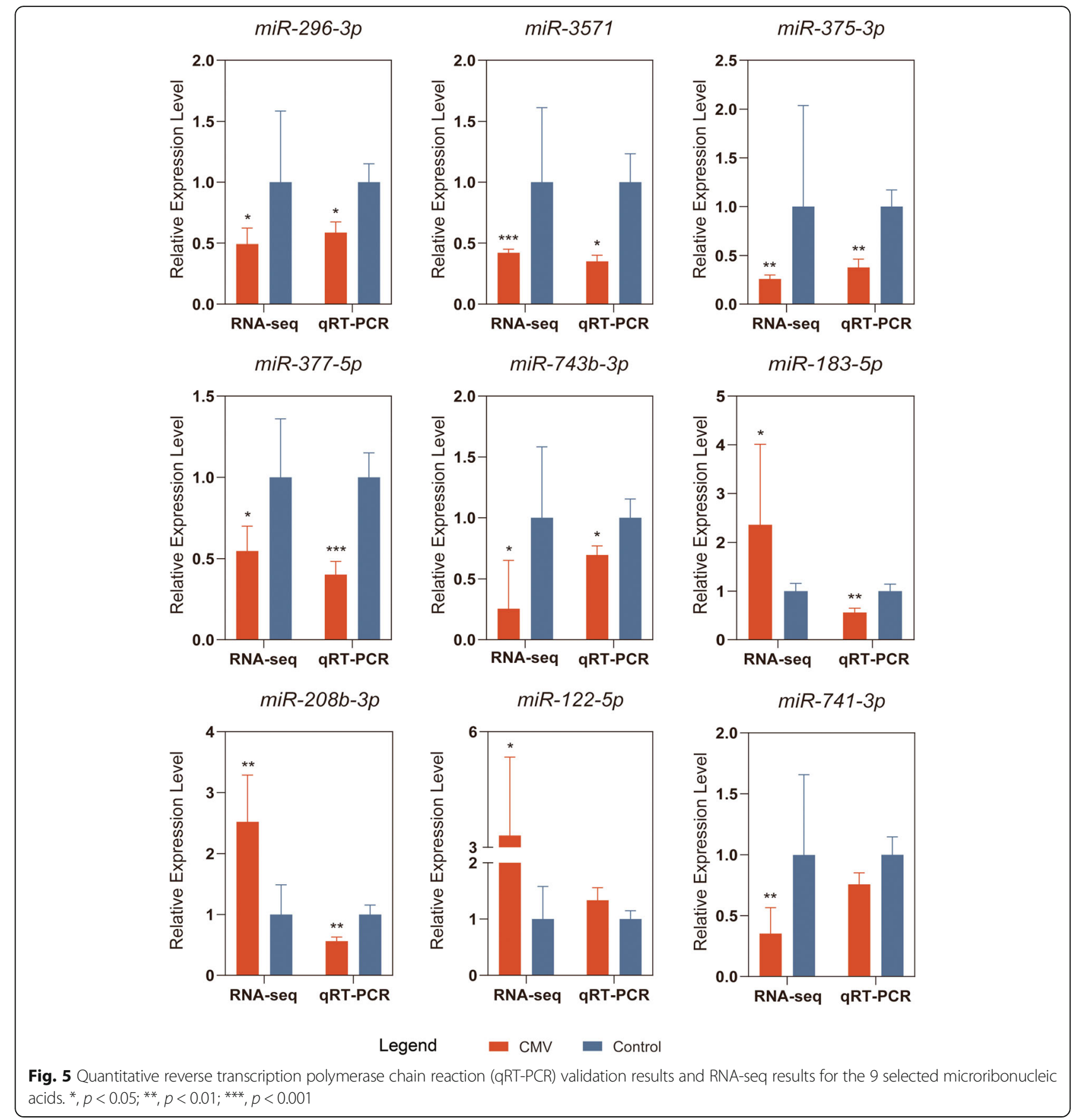

First, we showed that miRNA expression changed after $\mathrm{CMV}$, and these changes may reveal the physiological and pathophysiological processes occurring after CMV and may offer potential therapeutic targets for VIDD. We identified a total of 38 DE miRNAs in diaphragm tissue after CMV, among which 22 were upregulated and 16 were downregulated. The miRNAs and their Fold Change level were listed in Table 1. They might participate in the onset, progression, and development of VIDD. To our knowledge, only a few previous studies have illustrated the involvement of various miRNAs from different sources in VIDD. For example, Moroz, $\mathrm{N}$., et al. [12] reported that some autophagy-related regulated miRNAs, such as miR-106b, miR-20a, miR-101a, miR-376, miR-204 and miR-93, show lower expression in diaphragm tissue during mechanical ventilation. In other diseases, the downregulation of miR-1, miR-133, miR-486 and miR-206 has been observed in the diaphragms and limb muscles of mice with lung cancer cachexia [13]. The miRNA expression pattern of their 


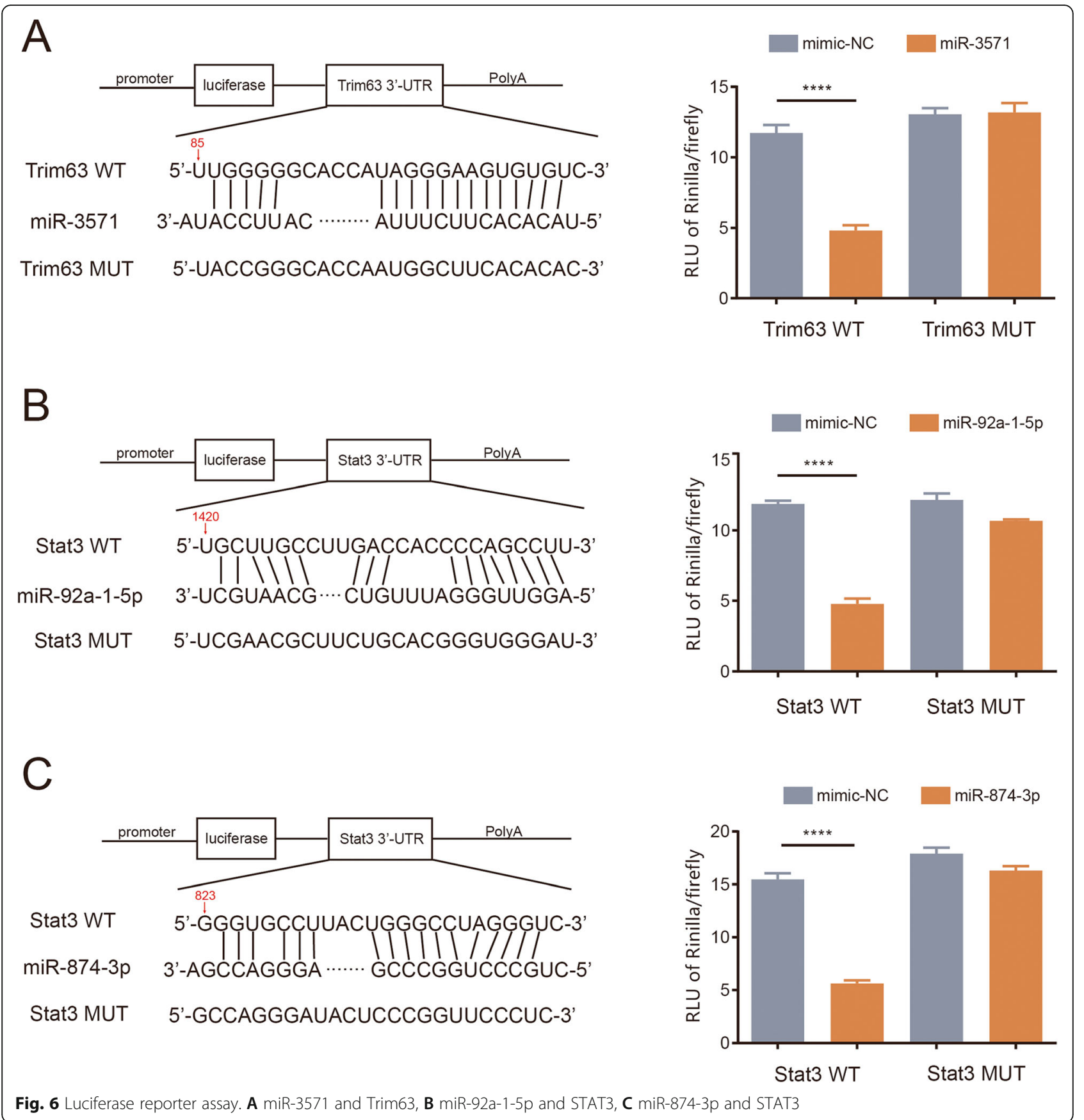

research was different from ours. We propose that this discrepancy may be due to sample and/or disease disparities, the miRNA expression alteration in induced diaphragm might be regulated by experimental conditions or species difference.

Several DE miRNAs that we identified have been reported in previous studies. For instance, miR-122-5p was up-regulated in our study, it has been reported to participate in regulating glioma [14, 15], hepatocellular carcinoma [16-20], myocardial injury [21], and osteoarthritis [22]. It has also been reported in studies of diseases of muscle dysfunction or degeneration, such as in normal skeletal muscle. The downregulation of miR-122-5p has been found in skeletal muscle fibrosis, and miR-122-5p overexpression has been shown to retard the fibrotic process by targeting the TGF- $\beta /$ Smad signaling pathway [23]. Another study showed a similar result: miR-122-5p may restore myogenesis [24]. These combined results show the potential therapeutic effects of this miRNA in translational studies. Additionally, miR-296-3p was downregulated in our study, it has been reported in several types of tumors, such as neurofibromatosis [25], lung 
adenocarcinoma [26], and prostate cancer [27]. As well as miR-375-3p and miR-874-3p, miR-375-3p was found to suppress tumorigenesis by targeting YAP1 and SP1 in colorectal cancer cells [28], and alleviate the severity of inflammation through targeting YAP1/LEKTI pathway in $\mathrm{HaCaT}$ cells [29], miR-874-3p was found as an antioncomir in esophageal squamous cell carcinoma via targeting STAT3 [30]. As to the down-regulated miR-3571, it was reported in associated with selenium deficiencyinduced cardiac dysfunction [31]. These miRNAs have not been previously reported to be associated with diaphragm dysfunction to our knowledge. However, in these diseases, the target genes they regulate are also reported in diaphragm function, or the similarity of some disease with VIDD (cardiac dysfunction), suggesting that these miRNAs may also have therapeutic effect on VIDD. Thus, our sequencing results may offer some novel findings.

To acquire insight into the prospective functions of the DE miRNAs, we performed KEGG pathway analyses to reveal the roles of the differentially expressed miRNAs in several biological pathways. Among these pathways, the MAPK signaling pathway and FoxO signaling pathway were the only two pathways that were correlated with environmental information processing and signal transduction. Based on the results of KEGG library database search [32], we constructed a pathway relationship network. This network showed the connections of the 20 most enriched signaling pathways, from which we found that the MAPK signaling pathway seemed to be the most important upstream signaling pathway, and that the FoxO signaling pathway was an important downstream pathway of MAPK. The autophagy-animal pathway was also identified. Several studies have shown the roles and prospective therapeutic effects of activating the FoxO signaling pathway [33] and autophagy [11, 34] in VIDD and have determined the underlying mechanisms.

The MAPK signaling pathway has been fully elucidated for quite some time; it is composed of a group of serine/ threonine protein kinases involved in cellular signal transduction that regulate many cellular functions and affect many human diseases [35]. Previous studies have proven the effects of MAPK signaling on tumor metastasis [36], osteoarthritis [37], traumatic brain injury [38], and certain inflammatory diseases [39]. For example, MAPK signaling is activated in the diaphragm tissue of mice with $\mathrm{Du}$ chenne muscular dystrophy [40]. In a model of nutritional deprivation-induced cachexia and muscle atrophy, the phosphorylation of MAPK/ERK was shown to be significantly downregulated in the diaphragm [41]. Another study also showed that MAPK/p38 and MK2 were associated with limb and diaphragm muscles [42]. Regarding other diseases, research on osteonecrosis showed that exosomes carrying overexpressed miR-122-5p regulated the
MAPK signaling pathway in the femoral head [43]. Other studies showed that the forced expression of miR-122-5p induced MAPK signaling in breast cancer [44]; that miR200a-5p regulated Se deficiency-induced myocardial necroptosis, which was hypothesized to be mediated by MAPK [45]; and that miR-200c-3p inhibited HMGB3, which can activate MAPK signaling, in glioblastoma [46]. This evidence collectively shows that several miRNAs identified in our study are related to the MAPK signaling pathway.

Based on previous studies [47], we selected several VIDD-related genes(e.g., Trim63 and STAT3). It is well established that Trim63 is upregulated in rats with VIDD after CMV [48], and JAK-STAT3 signaling is also altered [49]. On the basis of database searches, we speculated that they were regulated by several DE miRNAs found in our miRNA-seq results. In particular, STAT3 was regulated by miR-92a-1-5p and miR-874-3p, and Trim63 was regulated by miR-3571. Luciferase reporter assays were performed to verify the link between miRNAs and these genes. The results showed that miR-3571 can negatively regulate Trim63 and that miR-92a-1-5p and miR-874-3p can negatively regulate STAT3. Therefore, we hypothesized that these miRNAs may serve as critical targets in VIDD pathogenesis and that their upregulation may be a novel potential therapy for VIDD, giving rise to the opportunity for translation into human populations.

Finally, we predicted miRNA and targeted mRNA network according to the database and bioinformatic analysis criteria (Table S5) and constructed a miRNAmRNA network (Figure S3) by Cytoscape (v3.6.0). Briefly, the results showed that miR-296-3p, miR-147, miR-200c-3p and miR-877 regulated relatively few mRNAs, whereas miR-3571, miR-743b-3p and miR-96$5 p$ regulated more mRNAs. According to this analysis, the roles of these miRNAs and related mRNAs in VIDD may merit further research.

\section{Conclusions}

In the present study, we identified a series of DE miRNAs in diaphragm tissue after CMV-induced diaphragm dysfunction for the first time; these miRNAs may be linked to physiological and pathological processes during VIDD. We also estimated the potential roles of signaling pathways and miRNA-mRNA interactions. This study showed that miRNAs in diaphragm tissue are altered after VIDD, which implies that they may merit consideration as therapeutic targets in VIDD.

\section{Methods \\ Animals}

SPF-level adult male Wistar rats were obtained from Charles River Laboratories (Beijing, China); their weight 
varied from 450 to $550 \mathrm{~g}$. All animal studies were performed in the Bio-Safety Level III Laboratory of Wuhan University (Wuhan, Hubei, China). Animal experiments were approved by the Animal Experiment Center and the ethics committee of Zhongnan Hospital of Wuhan University and were conducted following the National Institutes of Health Guide for the Care and Use of Laboratory Animals. The rats were housed in dedicated cages under controlled conditions (temperature: $25{ }^{\circ} \mathrm{C} \pm$ $2{ }^{\circ} \mathrm{C}$; relative humidity: $50 \% \pm 5 \%$ ) with a $12: 12$ lightdark cycle. Water and food were provided ad libitum.

\section{Controlled mechanical ventilation (CMV) model and ventilator-induced diaphragm dysfunction (VIDD)}

Eighteen animals were randomly divided into two groups by using a table of random numbers: (1) a control group $(n=9)$, in which animals underwent a sham operation and were not ventilated; and (2) the CMV group $(n=9)$, in which animals received CMV for $12 \mathrm{~h}$. The animal model of mechanical ventilation was established in accordance with a previous study [50]. Briefly, animals were anaesthetized with sodium pentobarbital (40 mg/kg, ip.). After successful anaesthetization, rats were fixed on a recirculating heating blanket. They were tracheostomized and then connected to a volume-driven small animal ventilator (VentElite, USA). The ventilator parameters were set as follows: the respiratory rate (RR) was set at 55 to $60 \mathrm{rpm}$, and the tidal volume (TV) was set at $5 \mathrm{~mL} /$ $\mathrm{kg}$ body weight. The breathing air of the rats was humidified with sterilized saline and enriched with oxygen. Arterial blood gas analysis was conducted every $2 \mathrm{~h}$ during ventilation to maintain at a $\mathrm{PaCO}_{2}$ be-

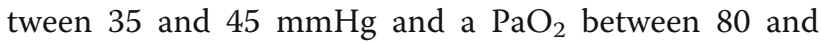
$100 \mathrm{mmHg}$ during the entire $12 \mathrm{~h}$ ventilation period to avoid ventilator-induced systemic hypoxia. Blood pressure (BP) and heart rate (HR) measured at the tail artery by tail cuff plethysmography (BP-2010 Series Blood Pressure Metre, Softron, Japan) were recorded in real time. We selected and cannulated the right jugular veins of the rats for the continuous infusion of normal sterilized saline (Baxter, Deerfield, IL) $(1 \mathrm{~mL} / \mathrm{kg} / \mathrm{h})$ and pentobarbital sodium $(10 \mathrm{mg} / \mathrm{kg} / \mathrm{h})$ with an electric pump. The body temperature of the rats was maintained at $37{ }^{\circ} \mathrm{C}$ during the study by external warming through a homeothermic blanket system [47]. The rats were conventionally euthanized by the intraperitoneal injection of an overdose of pentobarbital sodium $(100 \mathrm{mg} / \mathrm{kg})$ before the diaphragm was removed. Among all the samples, eight (four from the CMV group and four from the control group) were used for miRNA-seq, and ten (five from the CMV group and five from the control group) were selected for qRT-PCR validation.

\section{MicroRNA sequencing and subsequent bioinformatic analysis}

Total RNA was extracted from diaphragm tissue samples using a miRNeasy Mini Kit (Qiagen, Cat. No. 217004). RNA integrity was assessed using an Agilent 2100 Bioanalyzer (Agilent Technologies, USA). After this assessment, the miRNA samples with an RNA integrity number (RIN) $\geq 7$ were selected for subsequent analysis. TruSeq Small RNA Sample Prep Kit (Cat. No. RS-2000012, Illumina, USA.) was used for library construction. Small RNA sequencing and analysis were carried out by OE Biotech Co., Ltd. (Shanghai, China). The basic reads were transformed into raw data by base calling, and their sequences were obtained on the Illumina HiSeq 4000 sequencer platform. Low-quality reads were filtered. Reads with 5' primer contamination and poly (A) tails and those without 3 ' adapters were removed. In addition, reads without insert tags and those that were shorter than $15 \mathrm{nt}$ or longer than $41 \mathrm{nt}$ in the raw data were filtered to obtain the clean reads. The minimal read count was set as 1 .

First, the clean reads were used for length distribution analysis in the reference genome. These clean reads were aligned, subjected to BLAST database analysis [51], and subjected to searches against the Rfam v.10.1 (http:// www.sanger.ac.uk/software/Rfam) [52, 53] and GenBank databases (http://www.ncbi.nlm.nih.gov/genbank/). The noncoding RNAs were annotated as tRNAs, rRNAs, small nuclear RNAs (snRNAs), and small nucleolar RNAs (snoRNAs), and were excluded from the analysis. Known miRNAs were identified by alignment against the miRBase v.22.1 database (http://www.mirbase.org/) with Bowtie (v1.1.1) [53], and their expression levels were normalized by the transcript per million (TPM) method, according to the formula $\mathrm{N} / \mathrm{M}^{*} 10^{6}$, where $\mathrm{N}$ presents the reads for each miRNA, and $M$ presents the reads in each sample. Unannotated small RNAs were analysed with miRDeep2 [54] to predict the structure of novel miRNAs, based on the pre-hairpin structure and the criteria of the miRbase database.

DE miRNAs were identified as those meeting the threshold of a $p$ value $<0.05$ and a fold change $>2.0$. The fold change and $p$ values were calculated with the DEG algorithm (R, DESeq package) [55]. The target mRNAs of DE miRNAs were predicted by using the following databases: miRDB (http://mirdb.org), TargetScan (http:// www.targetscan.org) and Diana (http://diana.imis. athena-innovation.gr/DianaTools), and an intersection of all the target genes listed in the databases was selected.

GO (http://www.geneontology.org) enrichment and KEGG (http://www.genome.jp/kegg) pathway enrichment analyses of the DE miRNA-targeted genes were calculated based on the hypergeometric distribution ( $\mathrm{R}$, stats package) [56]. 


\section{qRT-PCR analysis}

We performed qRT-PCR to prove the accuracy of the miRNA-seq data. RNA samples were reverse transcribed into cDNA using a Mir-X miRNA First Strand Synthesis Kit (638313, TaKaRa, Osaka, Japan). Briefly, RNA samples were diluted in RNAse-free DEPC water and mixed with $\mathrm{mRQ}$ buffer and mRQ enzyme. Then they were incubated for $1 \mathrm{~h}$ at $37^{\circ} \mathrm{C}$, followed by $85{ }^{\circ} \mathrm{C}$ for $5 \mathrm{~min}$ to inactivate the enzymes on a thermal cycler. After reaction termination, the cDNA samples were diluted with a 9-fold volume of DEPC water. Real-time PCR was conducted using TB Green Premix Ex Taq II (Tli RNaseH Plus) (RR820A, TaKaRa, Japan). First, cDNA samples, primers, and DEPC water were mixed in 96-well plates, and qRT-PCR was performed on a CFX Connect RealTime PCR System (Bio-Rad; CFX Maestro 1.0 software). The thermal cycling program was set as follows: $95{ }^{\circ} \mathrm{C}$, $30 \mathrm{~s} ; 45$ PCR cycles $\left(95^{\circ} \mathrm{C}, 5 \mathrm{~s} ; 60^{\circ} \mathrm{C}, 30 \mathrm{~s}\right.$ [plate read]); and $95{ }^{\circ} \mathrm{C}, 10 \mathrm{~s}$, with melt curve analysis at the end of the program. The sequences of the primers used in this experiment are shown in Table S1.

\section{Luciferase reporter assay}

We selected the trim63 and STAT3 genes, which have been widely reported in VIDD studies, and the identified DE miRNAs for which not reported in VIDD yet. The miRNA-mRNA connections were selected according to the TargetScan database (http:// www.targetscan.org) and previous studies. The links between miR-3571 and Trim63, miR-92a-1-5p and STAT3, and miR-874-3p and STAT3 were determined, and a luciferase reporter assay was conducted for verification. Briefly, the 3'-UTRs of Trim63 and STAT3 with putative miRNA binding sequences (Rat Genome Database, https://rgd.mcw.edu) [WT (wild type) or MUT (mutant type)] were cloned, and the base sequences were confirmed by sequencing. The plasmid sequences carried in the vector were consistent with the expected sequences, confirming the successful construction of the vectors. The psiCHECK-2 vector (Uptbio, China) was double digested, and the target fragment was linked to the linearized vector at the insertion sites of XhoI and NotI. Lipofectamine 3000 (Thermo Fisher Scientific) was used to cotransfect the resulting constructs $(0.8 \mu \mathrm{g})$ with mimic-NC, miR-3571, miR-874-3p, or miR-92a-1-5p (each 100 nM) into HEK293 reporter cells (Procell, Wuhan, China) plated in 24-well plates. Cells were incubated at $37{ }^{\circ} \mathrm{C}$ for $24 \mathrm{~h}$, and the relative light unit (RLU) values of firefly luciferase and Renilla luciferase were subsequently detected and recorded with a Luminometer (Lux-T020, BLT). The ratio of Renilla luciferase to firefly luciferase in each group was calculated.

\section{Statistical analysis}

The statistical analyses were performed in SPSS 24.0. Data are expressed as the mean \pm SD format. Student's $t$ test was selected to evaluate differences between two groups in qRT-PCR. A $p$ value $<0.05$ was considered to indicate a significant difference.

\section{Abbreviations \\ CMV: Controlled mechanical ventilation; VIDD: Ventilator-induced diaphragm dysfunction; miRNA: Microribonucleic acid; RNA-seq: RNA-sequencing; DE: Differentially expressed; GO: Gene oncology; KEGG: Kyoto encyclopedia of gene and genomes; BP: Biological process; CC: Cellular component; MF: Molecular function; RT-qPCR: Reverse transcription-quantitative PCR}

\section{Supplementary Information}

The online version contains supplementary material available at https://doi. org/10.1186/s12864-021-07970-y.
Additional file 1: Table S1. Primers sequences used in this study.
Additional file 2: Table S2. Detailed information for differentially expressed (DE) miRNAs $\left(\left|\log _{2} \mathrm{FC}\right|>1\right.$ and $\left.p<0.05\right)$.

Additional file 3: Table S3. Detailed information for the differentially enriched $\mathrm{GO}$ term analysis.

Additional file 4: Table S4. Detailed information for the differentially enriched KEGG pathways.

Additional file 5: Table S5. Interaction of miRNAs and mRNAs.

Additional file 6: Figure S1. Novel miRNA categories.

Additional file 7: Figure S2. (A) GO level2 distribution of different miRNAs. (B) KEGG level2 distribution of different miRNAs.

Additional file 8: Figure $\mathbf{S 3}$. mi-mRNA relation network analysis. The blue circles indicate targeted mRNAs, and the red circles indicate the DE miRNAs related with these mRNAs. The size of the circles represents the number of connections.

\section{Acknowledgements}

The authors acknowledge the members of Emergency Center, Zhongnan Hospital of Wuhan University.

\section{Authors' contributions}

$P W, X Z$ and $Y Z$ conceived and designed the research plans. PW and $X Z$ established the CMV model and isolated RNA samples from the rat diaphragm. PW, GL, HM, XZ, and RL completed the data processing, normalization, and bioinformatics analyses. PW wrote the article with contributions from all the authors. $Y Z$ and $X Z$ proposed the conception of the work and substantively revised the manuscript. $Y Z, H M$ and $R L$ supervised and corrected the writing. All authors approved the final draft for submission.

\section{Funding}

This study was supported by the National Natural Science Foundation of China [grant number 81900097], the Emergency Response Project of Hubei Science and Technology Department [grant number 2020FCA023] and the Emergency Diagnostic and Therapeutic Center of Central China. The funders had no role in the study design, data collection and analysis, decision to publish, or preparation of the manuscript.

\section{Availability of data and materials}

All of the raw data have been uploaded to the Sequence Read Archive (SRA), and the accession number is SRX11758233-11758240. The data can be accessed at https://www.ncbi.nlm.nih.gov/bioproject/PRJNA753226. The miRNA database we refer to can be found at http://www.mirbase.org/cgibin/browse.pl?org=rno, the Genome Database can be found at ftp://ftp.ncbi. nlm.nih.gov/genomes/all/GCF/000/001/895/GCF_000001895.5_Rnor_6.0/ GCF_000001895.5_Rnor_6.0_genomic.fna.gz, and the mRNA Database can be found at ftp://ftp.ncbi.nlm.nih.gov/genomes/all/GCF/000/001/895/GCF_ 000001895.5_Rnor_6.0/GCF_000001895.5_Rnor_6.0_rna.fna.gz. All of the 
datasets referenced in this study can be obtained upon reasonable request to the corresponding authors.

\section{Declarations}

\section{Ethics approval and consent to participate}

All animal experimental procedures were approved by the Institutional

Animal Care and Use Committee of Wuhan University.

\section{Consent for publication}

Not applicable.

\section{Competing interests}

The authors declare that they have no competing interests.

\section{Author details}

'Emergency Center, Zhongnan Hospital of Wuhan University, 430071 Wuhan, China. ${ }^{2}$ Hubei Clinical Research Center for Emergency and Resuscitation, Zhongnan Hospital of Wuhan University, 430071 Wuhan, China. ${ }^{3}$ Department of Biological Repositories, Zhongnan Hospital of Wuhan University, 430071 Wuhan, China.

Received: 6 January 2021 Accepted: 2 September 2021

\section{Published online: 18 September 2021}

\section{References}

1. Zhang LJ, Ni SZ, Zhou XL, Zhao Y. Hemorrhagic Shock Sensitized the Diaphragm to Ventilator-Induced Dysfunction through the Activation of IL6/JAK/STAT Signaling-Mediated Autophagy in Rats. Mediators Inflamm. 2019;2019:3738409.

2. Gotts JE, Matthay MA. Sepsis: pathophysiology and clinical management. BMJ (Clinical research ed). 2016;353:i1585.

3. Mas A, Masip J. Noninvasive ventilation in acute respiratory failure. Int J Chronic Obstr Pulm Dis. 2014;9:837-52.

4. Ramachandran P, Swamy L, Kaul V, Agrawal AJC. A National Strategy for Ventilator and ICU Resource Allocation During the COVID-19 Pandemic. 2020.

5. Azuelos I, Jung B, Picard M, Liang F, Li T, Lemaire C, et al. Relationship between Autophagy and Ventilator-induced Diaphragmatic Dysfunction. Anesthesiology. 2015;122(6):1349-61.

6. Kavazis AN, Talbert EE, Smuder AJ, Hudson MB, Nelson WB, Powers SK Mechanical ventilation induces diaphragmatic mitochondrial dysfunction and increased oxidant production. Free Radic Biol Med. 2009;46(6):842-50.

7. Namuduri AV, Heras G, Mi J, Cacciani N, Hornaeus K, Konzer A, et al. A Proteomic Approach to Identify Alterations in the Small Ubiquitin-like Modifier (SUMO) Network during Controlled Mechanical Ventilation in Rat Diaphragm Muscle. Mol Cell Proteomics. 2017;16(6):1081-97.

8. Mankowski RT, Ahmed S, Beaver T, Dirain M, Han C, Hess P, et al. Intraoperative hemidiaphragm electrical stimulation reduces oxidative stress and upregulates autophagy in surgery patients undergoing mechanical ventilation: exploratory study. J Transl Med. 2016;14(1):305.

9. Borja-Gonzalez M, Casas-Martinez JC, McDonagh B, Goljanek-Whysall K. Inflamma-miR-21 Negatively Regulates Myogenesis during Ageing. Antioxidants (Basel). 2020;9(4). https://doi.org/10.3390/antiox9040345. Epub 29 April

10. Garros RF, Paul R, Connolly M, Lewis A, Garfield BE, Natanek SA, et al. MicroRNA-542 Promotes Mitochondrial Dysfunction and SMAD Activity and Is Elevated in Intensive Care Unit-acquired Weakness. Am J Respir Crit Care Med. 2017;196(11):1422-33.

11. Hudson MB, Rahnert JA, Zheng B, Woodworth-Hobbs ME, Franch HA, Price SR. miR-182 attenuates atrophy-related gene expression by targeting FoxO3 in skeletal muscle. Am J Physiol Cell Physiol. 2014;307(4):C314-9.

12. Moroz N, Maes K, Leduc-Gaudet JP, Goldberg P, Petrof BJ, Mayaki D, et al. Oxidants Regulated Diaphragm Proteolysis during Mechanical Ventilation in Rats. Anesthesiology. 2019;131(3):605-18.

13. Chacon-Cabrera A, Fermoselle C, Salmela I, Yelamos J, Barreiro E. MicroRNA expression and protein acetylation pattern in respiratory and limb muscles of Parp-1(-/-) and Parp-2(-/-) mice with lung cancer cachexia. Biochim Biophys Acta. 2015;1850(12):2530-43.
14. Liu N, Wang Z, Liu D, Xie P. HOXC13-AS-miR-122-5p-SATB1-C-Myc feedback loop promotes migration, invasion and EMT process in glioma. Onco Targets Ther. 2019;12:7165-73.

15. Li C, Hu G, Wei B, Wang L, Liu N. IncRNA LINC01494 Promotes Proliferation, Migration And Invasion In Glioma Through miR-122-5p/CCNG1 Axis. Onco Targets Ther. 2019;12:7655-62. https://doi.org/10.2147/OTT.S213345 Epub 02 Oct 2019.

16. Yang X, Yao B, Niu Y, Chen T, Mo H, Wang L, et al. Hypoxia-induced IncRNA EIF3J-AS1 accelerates hepatocellular carcinoma progression via targeting miR122-5p/CTNND2 axis. Biochem Biophys Res Commun. 2019;518(2):239-45.

17. Ma J, Li T, Han X, Yuan H. Knockdown of LncRNA ANRIL suppresses cell proliferation, metastasis, and invasion via regulating miR-122-5p expression in hepatocellular carcinoma. J Cancer Res Clin Oncol. 2018;144(2):205-14.

18. Yang $X$, Sun L, Wang L, Yao B, Mo H, Yang W. LncRNA SNHG7 accelerates the proliferation, migration and invasion of hepatocellular carcinoma cells via regulating miR-122-5p and RPL4. Biomed Pharmacother. 2019;118: 109386.

19. Liang Y, Zhang D, Zheng T, Yang G, Wang J, Meng F, et al. IncRNA-SOX2OT promotes hepatocellular carcinoma invasion and metastasis through miR122-5p-mediated activation of PKM2. Oncogenesis. 2020;9(5):54.

20. Gao J, Yin X, Yu X, Dai C, Zhou F. Long noncoding LINC01551 promotes hepatocellular carcinoma cell proliferation, migration, and invasion by acting as a competing endogenous RNA of microRNA-122-5p to regulate ADAM10 expression. J Cell Biochem. 2019;120(10):16393-407.

21. Peng H, Luo Y, Ying Y. IncRNA XIST attenuates hypoxia-induced $\mathrm{H} 9 \mathrm{c} 2$ cardiomyocyte injury by targeting the miR-122-5p/FOXP2 axis. Mol Cell Probes. 2020;50:101500.

22. Li X, Huang TL, Zhang GD, Jiang JT, Guo PY. LncRNA ANRIL impacts the progress of osteoarthritis via regulating proliferation and apoptosis of osteoarthritis synoviocytes. Eur Rev Med Pharmacol Sci. 2019;23(22):9729-37.

23. Sun Y, Wang H, Li Y, Liu S, Chen J, Ying H. miR-24 and miR-122 Negatively Regulate the Transforming Growth Factor-beta/Smad Signaling Pathway in Skeletal Muscle Fibrosis. Mol Ther Nucleic Acids. 2018;11:528-37.

24. Ding Z, Lin J, Sun Y, Cong S, Liu S, Zhang Y, et al. miR-122-5p negatively regulates the transforming growth factor-beta/Smad signaling pathway in skeletal muscle myogenesis. Cell Biochem Funct. 2020;38(2):231-8.

25. Lee H, Hwang SJ, Kim HR, Shin $\mathrm{CH}$, Choi KH, Joung JG, et al. Neurofibromatosis 2 (NF2) controls the invasiveness of glioblastoma through YAP-dependent expression of CYR61/CCN1 and miR-296-3p. Biochim Biophys Acta. 2016;1859(4):599-611.

26. Fu Q, Song X, Liu Z, Deng X, Luo R, Ge C, et al. miRomics and Proteomics Reveal a miR-296-3p/PRKCA/FAK/Ras/c-Myc Feedback Loop Modulated by HDGF/DDX5/beta-catenin Complex in Lung Adenocarcinoma. Clin Cancer Res. 2017;23(20):6336-50.

27. Liu X, Chen Q, Yan J, Wang Y, Zhu C, Chen C, et al. MiRNA-296-3p-ICAM-1 axis promotes metastasis of prostate cancer by possible enhancing survival of natural killer cell-resistant circulating tumour cells. Cell Death Dis. 2013;4: e928.

28. Xu X, Chen X, Xu M, Liu X, Pan B, Qin J, et al. miR-375-3p suppresses tumorigenesis and partially reverses chemoresistance by targeting YAP1 and SP1 in colorectal cancer cells. Aging. 2019;11(18):7357-85.

29. Cheng S, Di Z, Hirman AR, Zheng H, Duo L, Zhai Q, et al. MiR-375-3p alleviates the severity of inflammation through targeting YAP1/LEKTI pathway in HaCaT cells. Biosci Biotechnol Biochem. 2020;84(10):2005-13.

30. Yuan RB, Zhang SH, He Y, Zhang XY, Zhang YB. MiR-874-3p is an independent prognostic factor and functions as an anti-oncomir in esophageal squamous cell carcinoma via targeting STAT3. Eur Rev Med Pharmacol Sci. 2018;22(21):7265-73.

31. Xing Y, Liu Z, Yang G, Gao D, Niu X. MicroRNA expression profiles in rats with selenium deficiency and the possible role of the Wnt/beta-catenin signaling pathway in cardiac dysfunction. Int J Mol Med. 2015;35(1):143-52.

32. Zhao RT, Zhou J, Dong XL, Bi CW, Jiang RC, Dong JF, et al. Circular Ribonucleic Acid Expression Alteration in Exosomes from the Brain Extracellular Space after Traumatic Brain Injury in Mice. J Neurotrauma. 2018; 35(17):2056-66.

33. Smuder AJ, Sollanek KJ, Min K, Nelson WB, Powers SK. Inhibition of forkhead boxO-specific transcription prevents mechanical ventilation-induced diaphragm dysfunction. Crit Care Med. 2015;43(5):e133-42.

34. Sabater-Arcis M, Bargiela A, Furling D, Artero R. miR-7 Restores Phenotypes in Myotonic Dystrophy Muscle Cells by Repressing Hyperactivated Autophagy. Mol Ther Nucleic Acids. 2020;19:278-92. 
35. Seger R, Krebs EG. The MAPK signaling cascade. FASEB J. 1995;9(9):726-35.

36. Fliedner SM, Engel T, Lendvai NK, Shankavaram U, Nolting S, Wesley R, et al. Anti-cancer potential of MAPK pathway inhibition in paragangliomas-effect of different statins on mouse pheochromocytoma cells. PLoS One. 2014;9(5): e97712.

37. Wang P, Mao Z, Pan Q, Lu R, Huang X, Shang X, et al. Histone deacetylase-4 and histone deacetylase-8 regulate interleukin-1 beta-induced cartilage catabolic degradation through MAPKJJNK and ERK pathways. Int J Mol Med. 2018;41(4):2117-27.

38. Li J, Wang M, Chen X. Long non-coding RNA UCA1 modulates cell proliferation and apoptosis by regulating miR-296-3p/Myc axis in acute myeloid leukemia. Cell Cycle. 2020;19(12):1454-65.

39. Ismail HM, Yamamoto K, Vincent TL, Nagase H, Troeberg L, Saklatvala J. Interleukin-1 Acts via the JNK-2 Signaling Pathway to Induce Aggrecan Degradation by Human Chondrocytes. Arthritis Rheumatol. 2015;67(7):1826-36.

40. Dogra C, Srivastava DS, Kumar A. Protein-DNA array-based identification of transcription factor activities differentially regulated in skeletal muscle of normal and dystrophin-deficient mdx mice. Mol Cell Biochem. 2008;312(12):17-24.

41. Lewis MI, Bodine SC, Kamangar N, Xu X, Da X, Fournier M. Effect of severe short-term malnutrition on diaphragm muscle signal transduction pathways influencing protein turnover. J Appl Physiol (1985). 2006;100(6):1799-806.

42. Norrby M, Tagerud S. Mitogen-activated protein kinase-activated protein kinase 2 (MK2) in skeletal muscle atrophy and hypertrophy. J Cell Physiol. 2010;223(1):194-201.

43. Liao W, Ning Y, Xu HJ, Zou WZ, Hu J, Liu XZ, et al. BMSC-derived exosomes carrying microRNA-122-5p promote proliferation of osteoblasts in osteonecrosis of the femoral head. Clin Sci (Lond). 2019;133(18):1955-75.

44. Wang Z, Wang X. miR-122-5p promotes aggression and epithelialmesenchymal transition in triple-negative breast cancer by suppressing charged multivesicular body protein 3 through mitogen-activated protein kinase signaling. J Cell Physiol. 2020;235(3):2825-35.

45. Yang T, Cao C, Yang J, Liu T, Lei XG, Zhang Z, et al. miR-200a-5p regulates myocardial necroptosis induced by Se deficiency via targeting RNF11. Redox Biol. 2018;15:159-69.

46. Liu J, Wang L, Li X. HMGB3 promotes the proliferation and metastasis of glioblastoma and is negatively regulated by miR-200b-3p and miR-200c-3p. Cell Biochem Funct. 2018;36(7):357-65.

47. Zhou XL, Wei XJ, Li SP, Liu RN, Yu MX, Zhao Y. Interactions between Cytosolic Phospholipase A2 Activation and Mitochondrial Reactive Oxygen Species Production in the Development of Ventilator-Induced Diaphragm Dysfunction. Oxid Med Cell Longev. 2019;2019:2561929.

48. Zhou XL, Wei XJ, Li SP, Ma HL, Zhao Y. Lung-protective ventilation worsens ventilator-induced diaphragm atrophy and weakness. Respir Res. 2020;21 (1):16.

49. Tang H, Smith IJ, Hussain SN, Goldberg P, Lee M, Sugiarto S, et al. The JAKSTAT pathway is critical in ventilator-induced diaphragm dysfunction. Mol Med (Cambridge Mass). 2015;20(1):579-89.

50. Davis RT 3rd, Bruells CS, Stabley JN, McCullough DJ, Powers SK, Behnke BJ. Mechanical ventilation reduces rat diaphragm blood flow and impairs oxygen delivery and uptake. Crit Care Med. 2012;40(10):2858-66.

51. Altschul SF, Gish W, Miller W, Myers EW, Lipman DJ. Basic local alignment search tool. J Mol Biol. 1990;215(3):403-10.

52. Griffiths-Jones S, Bateman A, Marshall M, Khanna A, Eddy SR. Rfam: an RNA family database. Nucleic Acids Res. 2003;31 (1):439-41.

53. Griffiths-Jones S, Saini HK, van Dongen S, Enright AJ. miRBase: tools for microRNA genomics. Nucleic Acids Res. 2008;36(Database issue):D154-8.

54. Friedlander MR, Mackowiak SD, Li N, Chen W, Rajewsky N. miRDeep2 accurately identifies known and hundreds of novel microRNA genes in seven animal clades. Nucleic Acids Res. 2012;40(1):37-52

55. Huber SAW. Differential expression analysis for sequence count data. Genome Biol. 2010;11:R106

56. Team RC. R: A language and environment for statistical computing. $R$ Foundation for Statistical Computing, Vienna, Austria https://www.R-project. org/. 2018.

\section{Publisher's Note}

Springer Nature remains neutral with regard to jurisdictional claims in published maps and institutional affiliations.

\section{Ready to submit your research? Choose BMC and benefit from:}

- fast, convenient online submission

- thorough peer review by experienced researchers in your field

- rapid publication on acceptance

- support for research data, including large and complex data types

- gold Open Access which fosters wider collaboration and increased citations

- maximum visibility for your research: over $100 \mathrm{M}$ website views per year

At BMC, research is always in progress.

Learn more biomedcentral.com/submissions 